\title{
The relationship between self-reported and registry-based data on use of psychoactive medications in postmenopausal women
}

\author{
Päivi H Rauma ${ }^{1,2^{*}}$, Heli Koivumaa-Honkanen ${ }^{3,4,5}$, Heikki Kröger ${ }^{2,6}$, Marjo T Tuppurainen ${ }^{2,7}$, Jussi Kauhanen ${ }^{8}$ \\ and Risto J Honkanen ${ }^{2,8}$
}

\begin{abstract}
Background: Self-report is commonly used as a source of information on the use of medicine. The aim of this study was to investigate the relationship between self-reported and register-based information on the use of psychoactive medication, especially in respect to antidepressants, and reasons of non-reporting.

Methods: Study subjects $(n=11,031)$ originated from a population-based cohort of postmenopausal women born in 1932-41 from Eastern Finland who responded to a postal enquiry in 1999. Self-reported currently used prescribed medications were compared to the National prescription register data. Diuretics served as a reference for psychoactive medications.

Results: Only 44\% out of 1,638 women reported their use of psychoactive medication when compared to the prescription register within a 4-month time window preceding their response to enquiry. Altogether, $55 \%$ out of 777 women reported their use of antidepressants and $29 \%$ out of 861 reported their use of other psychoactive medications. In comparison 83\% reported their use of diuretics. After excluding the occasional use, an increase in sensitivity by approximately 10 percentage points was seen regardless of the group of psychoactive medication. High use and history of work disability pension due to psychiatric cause were associated with a much higher likelihood of reporting psychoactive medication use (for antidepressants $70 \%$ and $81 \%$, respectively).

Conclusions: For research purposes, self-reported current use of psychoactive medication seems to be a sufficient indicator for regular use of antidepressants or in respect of use of any psychoactive medication, for subjects with severe psychiatric disease.
\end{abstract}

Keywords: Antidepressants, Menopause, Psychoactive drugs, Registers, Self-report, Validation studies

\section{Background}

Self-report is commonly used as a source for information regarding medication exposure in epidemiological studies. This type of data is dependent on memory and maybe vulnerable to recall bias, but also unwillingness to report may play a role. Thus, for valid results, accurate information or at least knowledge of the degree of misreports is needed. The validity of self-reported medication use can be assessed by comparing self-reports to

\footnotetext{
* Correspondence: pr.rauma@gmail.com

'Social Pharmacy, School of Pharmacy, Faculty of Health Sciences, University of Eastern Finland (UEF), P.O. Box 1627, 70211, Kuopio, Finland

'Bone and Cartilage Research Unit, Surgery, Institute of Clinical Medicine, UEF, Kuopio, Finland

Full list of author information is available at the end of the article
}

other data sources such as pharmacy data or medical records. Previous studies have investigated several different groups of medications [1-10]. For example, Klungel et al. [10] reported that approximately $70 \%$ of all medications currently used by patients with hypertension were correctly recalled in a self-administered questionnaire. However, the validity of self-reports has been found to vary extensively according to the type of medication taken [9,11-13]. The use of psychoactive medication has been shown to be less accurately reported than other medications $[9,11,13]$.

Depression is one of the leading causes of disease burden in the world and a major public health problem also among the elderly [14]. The occurrence of depression is

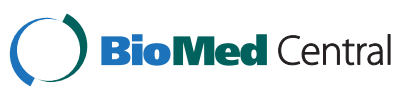


higher in women than men and its incidence peaks again after menopause [15]. There is limited validation data focusing solely [16-19] or among others [9,11-13,20-22] on the use of antidepressants or other psychoactive agents. Especially large cohort studies are rare [13,22]. Only few studies have investigated factors affecting accuracy of self-reported use of these medications [16,22]. Thus, more knowledge is needed regarding the extent and reasons associated with non-reporting of psychoactive medications, which could serve as a guide for future research.

The aim of the present study is to investigate among postmenopausal women from Eastern Finland, the agreement between self-reported use and registry-based data on purchased psychoactive medications, with particular reference to antidepressants. The causes for nonreports will also be investigated.

\section{Methods}

\section{Study design and subjects}

The study population $(n=11,031)$ for this crosssectional population-based study was formed as follows: in 1989, a baseline postal enquiry was sent to all the 14,220 female residents born in 1932-41 of Kuopio Province, Finland (OSTPRE Study) [23,24]. The 10-year follow-up enquiry in 1999 was sent to 12,562 women alive and with known address out of the 13,100 baseline respondents. This enquiry was returned by 11,537 women. Out of these 11,537 women, 11,031 women responded to the question determining current medication usage in 1999. The study has been approved by the ethics committee of Kuopio University Hospital. Informed consent of the study subjects was obtained by postal enquiry.

\section{Questionnaire data}

The study questionnaire included questions on marital status, residency (rural/city), height, weight, number of chronic health disorders, number of prescribed medications, use of hormone therapy (HT) (no/yes), life satisfaction (LS), physical activity (hours per week), smoking (no/yes) and consumption of beer, wine and distilled alcoholic beverages. The total use of alcohol was computed as grams (12 g/drink) per month and categorized (none/<360 g/ $\geq 360 \mathrm{~g}$ ). Body mass index (BMI) was calculated as weight $(\mathrm{kg}) /$ height $(\mathrm{m})$ squared and categorized $\left(<25 / 25-29 / \geq 30 \mathrm{~kg} / \mathrm{m}^{2}\right)$. LS was measured with a 4-item scale (range 4-20) [25-29]. It was used as a 3category variable: 4-6 (satisfied), 7-11 (intermediate) and 12-20 (dissatisfied) based on the standard deviation of the mean [27]. Education (4-category) was asked on a subsample $(n=3,222)$ who underwent bone densitometry. All these factors were tested as covariates in multivariate analyses.
Participants were asked current medication usage: "Are you at the moment under medication prescribed by a doctor? Specify the names of the medications." The reported medications were coded according to the Anatomical Therapeutic Chemical (ATC) code [30]. The ATC-categories investigated in the present study were: any psychoactive medications (N05, N06), antidepressants (N06A, N06CA) (with or without using other psychoactive medications) and other psychoactive medications (N05, N06B, N06CB, N06D) (without using antidepressants). In addition, "sole use of antidepressants" was also investigated. Purchasing of anti-dementia drugs (ATC: N06D) or psychostimulants/nootropics (N06B) during 2000-2005 according to National prescription register was used as memory variable (no/yes). Diuretics (C03), which are an independent, usual and regularly used medication group, were chosen to serve as a reference for psychoactive medications. Diuretics were used as follows: 1) any use (in tables), 2) together with psychoactive medication and 3) without psychoactive medication.

\section{Register data}

The study questionnaire data was linked with the National prescription register and the National Register for Work Disability Pensions by personal identification codes. The prescription register from the Social Insurance Institution of Finland (KELA) contains data regarding all reimbursed prescription medicines purchased in any pharmacy in Finland. It does not include information on 1) medication use in hospitals or community nursing homes, where medicines are included in the services; 2) medications which are not reimbursable such as small packages costing under a fixed deductible share (i.e. 8.41 $€$ in 1999) unless being a specially refunded medicine due to the selected chronic diseases including psychosis [31]. The defined daily doses (DDD) for medical products have been obtained from the Finnish Medicines Agency (FIMEA).

History of work disability pension up to the year 1996 (no/due to somatic cause/due to psychiatric cause) and medications purchased from a pharmacy within four or within 12 months before response to the enquiry in 1999 as DDD tertiles were used in the analysis. In Finland, only 3 months' refunded medication can be purchased from pharmacy at a time. Thus, a 4-month time window preceding the date of response to enquiry was chosen for prescription register data to ensure that a person would purchase a medication at least once (if used continuously) [19]. In order to explore the agreement of self-report, also a 12-month time window preceding the enquiry and a 4-month time window after the enquiry were applied. For loss analysis, the index date 
for women who did not return the questionnaire was the date the questionnaire was sent.

\section{Statistical analyses}

Statistical analyses were performed using the SPSS statistical package 17.0 for Windows (SPSS Inc., Chicago, IL, USA). Differences between study groups were examined with the Chi-squared test and the t-test for independent groups, or, in the case of variables not following a normal distribution, the non-parametric Mann-Whitney U-test. Agreement between the selfreported use and the National prescription register data as the golden standard were analyzed with sensitivity (i.e. recall rate or accuracy), specificity and Kappavalues (overall agreement).

To study factors affecting agreement of self-report and register data, logistic regression models were used. Only those women who according to the prescription register had purchased antidepressants or psychoactive medications were selected. Self-report (no/yes) was the outcome measure (i.e. dependent variable). Multivariate analysis was used to assess the independent effect of certain personal and health-related factors. Covariates were chosen into models if they were significantly associated with outcome. In the multivariate model 1, age, marital status, BMI, alcohol consumption and LS were covariates. In model 2, additional covariates included history of work disability pension up to the year 1996 and number of self-reported prescribed medications as well as amount of purchased psychoactive medications from the prescription register.

\section{Results}

\section{Characteristics}

According to the prescription register, 1,638 (14.8\%) of the 11,031 participants, had purchased psychoactive medications during the four months preceding the selfreport. A total of 777 (7.0\%) women had purchased antidepressants with or without other psychoactive medications, while 861 (7.8\%) had purchased other psychoactive medications than antidepressants. Out of the 777 antidepressant users, 393 (3.6\%) were "sole antidepressant users", while 384 women also used other psychoactive medications. Among these 384 women, antipsychotics were used by 123 women, anxiolytics by 198 women and hypnotics or sedatives by 184 women. In the entire group of users of other psychoactive medications $(n=861)$, antipsychotics were used by 147 women, anxiolytics by 315 and hypnotics or sedatives by 513 women. Users of psychoactive medications based on prescription register differed from non-users for all the study variables (Table 1). Users had more diseases and were less satisfied with their lives, while non-users were better off in terms of smoking and BMI, but not in terms of alcohol use. Of the entire study population, $22.7 \%$ had a history of work disability pension, this proportion being highest among users of antidepressants (43.2\%).

\section{Sensitivity between self-reported and prescription register data}

Self-reported use of medication was compared to the prescription register data within a 4-month time window preceding the response to enquiry (Tables 2 and 3). The sensitivity of self-reports was $44.2 \%$ for all psychoactive medication. It was higher for use of antidepressants (54.8\%) than for use of other psychoactive medications (29.0\%). For sole antidepressants users, the proportion was $50.1 \%$. Altogether, 66 women reported use of antidepressants and 152 women reported use of any psychoactive medication, though they had not purchased the medication within the 4-month time window preceding the self-report (Table 2). For the preceding 12 months prior to the self-report, these figures were 12 and 40 persons, respectively.

Self-reported use of diuretics covered a much higher percentage (83.2\%) of the register-based data than use of psychoactive medication. Users of psychoactive medication also reported their use of diuretics less accurately (76.0\%) than non-users $(85.1 \%)(P=0.001)$.

The sensitivity in use of psychoactive medication between the two data sources was better when only regular use was considered by taking into account medication purchased within four months both before and after the enquiry: the proportion of those reporting their use of medication was, thus, $55 \%$ for all psychoactive medications and $65 \%$ for antidepressants.

The exclusion of the low use tertile (i.e. occasional/irregular use) and the widening of the register data time window improved the sensitivity between self-report and register data. Thus, the sensitivity of self-reported regular use of antidepressants was $71.3 \%$, if the medication purchase precondition was four months before and after the response to enquiry. The proportion of false positive self-reports decreased from 13.4 to $2.5 \%$, if the time window was widened from 4 to 12 months before the response to enquiry.

Sensitivity and specificity of self-reports were 0.44 and 0.98 respectively, for psychoactive medication and 0.83 and 0.98 respectively for diuretics (Table 2). Kappavalues were 0.53 for all psychoactive medication, 0.65 for antidepressants, 0.30 for other psychoactive medications and 0.82 for diuretics (all $P<0.001$ ). Sensitivity of selfreported psychoactive medication increased markedly according to the amount of use (Table 3).

\section{Factors affecting sensitivity of self-report}

The amount of purchased psychoactive medications (DDDs/four months), history of work disability pension 
Table 1 Characteristics of study population $(n=11,031)$ by use of medication based on the National prescription register

\begin{tabular}{|c|c|c|c|c|c|c|c|c|c|c|c|c|}
\hline \multirow{2}{*}{$\frac{\text { Characteristic }}{\text { Continuous }}$} & \multicolumn{2}{|c|}{$\begin{array}{l}\text { All }(n= \\
11,031)\end{array}$} & \multicolumn{2}{|c|}{$\begin{array}{l}\text { No psychoactive } \\
\text { medication } \\
(n=9,393)\end{array}$} & \multicolumn{2}{|c|}{$\begin{array}{l}\text { Any psychoactive } \\
\text { medication users } \\
(n=1,638)\end{array}$} & \multicolumn{2}{|c|}{$\begin{array}{l}\text { Antidepressants } \\
\text { users }(n=777)\end{array}$} & \multicolumn{2}{|c|}{$\begin{array}{l}\text { Other psychoactive } \\
\text { medication users } \\
\text { without antidepressants } \\
(\mathrm{n}=861)\end{array}$} & \multicolumn{2}{|c|}{$\begin{array}{l}\text { Diuretics users } \\
(n=1,249)\end{array}$} \\
\hline & mean & $(\mathrm{SD})$ & mean & (SD) & mean & (SD) & mean & (SD) & mean & (SD) & mean & (SD) \\
\hline Age (years) & 62.3 & (2.9) & 62.2 & $(2.9)$ & 62.5 & $(3.0)^{* * *}$ & 62.3 & (3.0) & 62.7 & $(2.9)^{* * *}$ & 62.9 & $(2.9)^{* * *}$ \\
\hline BMI $\left(\mathrm{kg} / \mathrm{m}^{2}\right)$ & 27.6 & $(4.7)$ & 27.5 & (4.6) & 28.3 & $(5.2)^{* * *}$ & 28.4 & $(5.2)^{* * *}$ & 28.2 & $(5.2)^{* * *}$ & 30.5 & $(5.5)^{* * *}$ \\
\hline $\begin{array}{l}\text { Number of prescribed } \\
\text { medications }\end{array}$ & 2.1 & $(2.0)$ & 1.8 & (1.9) & 3.3 & $(2.2)^{* * *}$ & 3.5 & $(2.2)^{* * *}$ & 3.1 & $(2.2)^{* * *}$ & 4.0 & $(2.2)^{* * *}$ \\
\hline Number of diseases & 2.4 & (1.8) & 2.3 & (1.8) & 3.2 & $(2.0)^{* * *}$ & 3.2 & $(2.0)^{* * *}$ & 3.2 & $(1.9)^{* * *}$ & 3.5 & $(2.0)^{* * *}$ \\
\hline Self-rated $L S^{a}$ & 8.1 & $(2.8)$ & 7.9 & (2.6) & 9.4 & $(3.5)^{* * *}$ & 9.9 & $(3.6)^{* * *}$ & 9.0 & $(3.3)^{* * *}$ & 8.5 & $(3.1)^{* * *}$ \\
\hline Categorical & $n$ & (\%) & $n$ & (\%) & $n$ & (\%) & $n$ & $(\%)$ & $n$ & $(\%)$ & $n$ & $(\%)$ \\
\hline Disability pension ${ }^{c}$ & 2,500 & $(22.7)$ & 1,854 & $(19.7)$ & 646 & $(39.5) * * *$ & 328 & $(43.2) * * *$ & 318 & $(36.9)^{* * *}$ & 416 & $(33.3)^{* * *}$ \\
\hline HT use & 2,405 & $(21.8)$ & 2,010 & $(21.4)$ & 395 & $(24.1)^{*}$ & 215 & $(27.7)^{* * *}$ & 180 & (20.9) & 224 & $(17.9)^{* * *}$ \\
\hline Smoking & 908 & $(8.2)$ & 695 & (7.4) & 213 & $(13.0)^{* * *}$ & 90 & $(11.6) * * *$ & 123 & $(14.3)^{* * *}$ & 87 & $(7.0)$ \\
\hline Use of alcohol & 5,487 & $(51.4)$ & 4,749 & $(52.1)$ & 738 & $(47.0) * * *$ & 344 & $(46.3) * *$ & 394 & $(47.6)^{*}$ & 540 & $(45.6)^{* * *}$ \\
\hline
\end{tabular}

Abbreviations: $B M I$, body mass index; $H T$, hormone therapy; $S D$, standard deviation.

${ }^{*} P$ value $<0.05,{ }^{* *} P<0.005,{ }^{* * *} P \leq 0.001$ difference from non-users.

a Self-rated life satisfaction, range 4 to 20 , higher score indicating lower life satisfaction.

${ }^{\mathrm{b}}$ The group includes all diuretics users.

c History of work disability pension up to 1996.

due to psychiatric cause and the number of any selfreported prescribed medications as well as being unmarried were strongly associated with higher sensitivity between self-reported and register-based data regardless of type of medication (Table 3). Among users of any psychoactive medication also life dissatisfaction and higher BMI were associated with better sensitivity.

In multivariate models, greater alcohol consumption was associated with lower sensitivity for use of antidepressants (odds ratios, $\mathrm{OR}_{\text {model } 1}=0.39, P=0.045$ and $\mathrm{OR}_{\text {model2 }}=0.34, P=0.043$ ) (Table 4 ). In respect to marital status, those who are single had higher sensitivity than others (Tables 4 and 5). Higher number of any self- reported prescribed medication, higher use of antidepressants or any psychoactive medication according to prescription register and work disability pension due to psychiatric cause (model 2) were all associated independently with higher sensitivity, while work disability pension due to somatic cause was associated with lower sensitivity.

Among women using any psychoactive medication $(\mathrm{n}=$ 1,638) (Table 5), improved sensitivity between self-report and register-based data was associated with younger age (all models), but not with residence or physical activity nor was it related to education in the sample of 3,222 women, or to registry-based data on subsequent use of

Table 2 Comparison of self-reports and the National prescription register data for psychoactive medication and diuretics

\begin{tabular}{|c|c|c|c|c|c|c|c|c|}
\hline & \multicolumn{8}{|c|}{ Self-reported } \\
\hline & \multicolumn{2}{|c|}{ Any psychoactive medication } & \multicolumn{2}{|c|}{ Antidepressants } & \multicolumn{2}{|c|}{$\begin{array}{l}\text { Other psychoactive medication } \\
\text { without antidepressants }\end{array}$} & \multicolumn{2}{|c|}{ Diuretics ${ }^{b}$} \\
\hline & yes & no & yes & no & yes & no & yes & no \\
\hline \multicolumn{9}{|c|}{ Prescription register } \\
\hline Yes $^{a}$ & 724 & 914 & 426 & 351 & 250 & 611 & 1,039 & 210 \\
\hline No & 152 & 9,241 & 66 & 10,188 & 344 & 9,826 & 197 & 9,585 \\
\hline Sensitivity & 0.44 & & 0.55 & & 0.29 & & 0.83 & \\
\hline Specificity & 0.98 & & 0.99 & & 0.97 & & 0.98 & \\
\hline карра & $0.527^{*}$ & & $0.652^{*}$ & & $0.299^{*}$ & & $0.815^{*}$ & \\
\hline
\end{tabular}

* $P$ value $<0.001$.

${ }^{\text {a }}$ Purchased the given medication within four months before response to postal enquiry.

${ }^{b}$ The group includes all diuretics users. 
Table 3 Sensitivity (\%) of self-reported psychoactive medication use by characteristics of study subjects compared to the National prescription register data during the four months preceding the self-report

\begin{tabular}{|c|c|c|c|c|c|c|c|c|c|}
\hline \multicolumn{10}{|c|}{ Use of psychoactive medication according to the National prescription data } \\
\hline \multirow[b]{2}{*}{ Characteristics } & \multicolumn{3}{|l|}{ Any } & \multicolumn{3}{|c|}{ Antidepressant } & \multicolumn{3}{|c|}{ Other } \\
\hline & $\%$ & (n) & $P$ value $^{a}$ & $\%$ & (n) & $P$ value $^{\mathrm{a}}$ & $\%$ & (n) & $P$ value $^{\mathrm{a}}$ \\
\hline Self-reported use of psychoactive medication & 44.2 & $(724 / 1,638)$ & & 54.8 & $(426 / 777)$ & & 29.0 & $(250 / 861)$ & \\
\hline Age (years) & & & $<0.001$ & & & 0.254 & & & $<0.001$ \\
\hline $57-62$ & 49.3 & $(429 / 871)$ & & 56.6 & $(248 / 438)$ & & 36.0 & $(156 / 433)$ & \\
\hline $63-68$ & 38.5 & $(295 / 767)$ & & 52.5 & $(178 / 339)$ & & 22.0 & $(94 / 428)$ & \\
\hline Marital status & & & $<0.001$ & & & 0.021 & & & $<0.001$ \\
\hline Single & 64.3 & $(99 / 154)$ & & 70.0 & $(56 / 80)$ & & 47.3 & $(35 / 74)$ & \\
\hline Married / cohabitating & 42.3 & $(413 / 977)$ & & 54.8 & $(251 / 458)$ & & 26.2 & $(136 / 519)$ & \\
\hline Divorced & 50.0 & $(87 / 174)$ & & 51.7 & $(45 / 87)$ & & 40.2 & $(35 / 87)$ & \\
\hline Widoved & 36.7 & $(114 / 311)$ & & 48.9 & $(69 / 141)$ & & 22.9 & $(39 / 170)$ & \\
\hline Body mass index $\left(\mathrm{kg} / \mathrm{m}^{2}\right)$ & & & 0.011 & & & 0.195 & & & 0.115 \\
\hline$<25$ & 41.1 & $(170 / 414)$ & & 50.8 & $(93 / 183)$ & & 27.7 & $(64 / 231)$ & \\
\hline $25-29.99$ & 42.1 & $(262 / 623)$ & & 54.4 & $(162 / 298)$ & & 27.1 & $(88 / 325)$ & \\
\hline$\geq 30$ & 49.8 & $(240 / 482)$ & & 59.6 & $(137 / 230)$ & & 34.5 & $(87 / 252)$ & \\
\hline Alcohol consumption (g/month) & & & $<0.001$ & & & 0.075 & & & $<0.001$ \\
\hline None & 48.1 & $(400 / 832)$ & & 57.1 & $(228 / 399)$ & & 33.9 & $(147 / 433)$ & \\
\hline$<360$ & 38.0 & $(264 / 695)$ & & 51.3 & $(164 / 320)$ & & 21.9 & $(82 / 375)$ & \\
\hline$\geq 360$ & 51.2 & $(22 / 43)$ & & 37.5 & $(9 / 24)$ & & 63.2 & $(12 / 19)$ & \\
\hline Number of self-reported diseases & & & 0.911 & & & 0.365 & & & 0.670 \\
\hline 0 & 45.9 & $(34 / 74)$ & & 44.4 & $(16 / 36)$ & & 34.2 & $(13 / 38)$ & \\
\hline $1-2$ & 43.4 & $(258 / 594)$ & & 53.5 & $(154 / 288)$ & & 29.1 & $(89 / 306)$ & \\
\hline$\geq 3$ & 44.0 & $(410 / 931)$ & & 56.1 & $(245 / 437)$ & & 27.7 & $(137 / 494)$ & \\
\hline Smoking & & & 0.190 & & & 0.410 & & & 0.075 \\
\hline no & 43.6 & $(621 / 1,425)$ & & 54.3 & $(373 / 687)$ & & 27.9 & $(206 / 738)$ & \\
\hline yes & 48.4 & $(103 / 213)$ & & 58.9 & $(53 / 90)$ & & 35.8 & $(44 / 123)$ & \\
\hline HT use & & & 0.727 & & & 0.872 & & & 0.357 \\
\hline no & 44.0 & $(546 / 1,240)$ & & 55.2 & $(309 / 560)$ & & 29.9 & $(203 / 680)$ & \\
\hline yes & 45.1 & $(178 / 395)$ & & 54.4 & $(118 / 217)$ & & 26.1 & $(47 / 180)$ & \\
\hline Life satisfaction & & & 0.016 & & & 0.222 & & & 0.472 \\
\hline satisfied & 42.5 & $(102 / 240)$ & & 63.0 & $(58 / 92)$ & & 25.6 & $(53 / 207)$ & \\
\hline intermediate & 42.0 & $(402 / 958)$ & & 53.1 & $(229 / 431)$ & & 28.7 & $(142 / 494)$ & \\
\hline dissatisfied & 50.1 & $(212 / 423)$ & & 54.9 & $(134 / 244)$ & & 31.4 & $(45 / 143)$ & \\
\hline Number of self-reported prescribed medication & & & $<0.001$ & & & $<0.001$ & & & $<0.001$ \\
\hline 0 & 0.0 & $(0 / 110)$ & & 0.0 & $(0 / 35)$ & & 0.0 & $(0 / 75)$ & \\
\hline $1-2$ & 34.2 & $(202 / 590)$ & & 40.8 & $(115 / 282)$ & & 24.4 & $(75 / 308)$ & \\
\hline$\geq 3$ & 55.7 & $(522 / 938)$ & & 67.6 & $(311 / 460)$ & & 36.6 & $(175 / 478)$ & \\
\hline Amount of purchased medication ${ }^{b}$ & & & $<0.001$ & & & $<0.001$ & & & $<0.001$ \\
\hline low & 23.4 & $(120 / 513)$ & & 35.4 & $(85 / 240)$ & & 12.9 & $(40 / 309)$ & \\
\hline moderate & 42.5 & $(259 / 610)$ & & 56.8 & $(151 / 266)$ & & 39.6 & $(82 / 207)$ & \\
\hline high & 67.0 & $(345 / 515)$ & & 70.1 & $(190 / 271)$ & & 37.1 & $(128 / 345)$ & \\
\hline History of work disability pension & & & $<0.001$ & & & $<0.001$ & & & $<0.001$ \\
\hline no & 38.7 & $(384 / 992)$ & & 51.4 & $(231 / 449)$ & & 24.3 & $(132 / 543)$ & \\
\hline
\end{tabular}


Table 3 Sensitivity (\%) of self-reported psychoactive medication use by characteristics of study subjects compared to the National prescription register data during the four months preceding the self-report (Continued)

\begin{tabular}{lllllll}
\hline somatic cause & 39.1 & $(181 / 463)$ & 47.4 & $(99 / 209)$ & 25.6 & $(65 / 254)$ \\
psychiatric cause & 86.9 & $(159 / 183)$ & 80.7 & $(96 / 119)$ & 82.8 & $(53 / 64)$ \\
\hline
\end{tabular}

Abbreviations: $H T$, hormone therapy.

a $P$ value from the chi-square test between the subgroups.

b Tertile cutoffs for amount of use (DDD/4 months): psychoactive medication $<34$ (low), 34-100 (moderate), $>100$ (high), antidepressants $<33,33-99.9, \geq 100$, other psychoactive medication $<33,33-99.99, \geq 100$.

medication for dementia. However, only 92 women out of the entire study population had purchased anti-dementia medication in 2000-2005. Obesity was associated with better sensitivity in the use of any psychoactive medication (model 1), but the inclusion of the number of selfreported prescribed medications and history of work disability variables into the model totally abolished this association (Table 5).

Table 4 Factors related to the sensitivity of self-reported use of antidepressants compared to register-based data ( $n=777)$ by logistic models

\begin{tabular}{|c|c|c|c|c|c|c|c|c|c|}
\hline \multirow[b]{2}{*}{ Variables } & \multicolumn{3}{|c|}{ Univariate model } & \multicolumn{3}{|c|}{ Multivariate model $1^{c}(n=679)$} & \multicolumn{3}{|c|}{ Multivariate model $2^{c}(n=679)$} \\
\hline & $\overline{\mathrm{OR}}$ & $95 \% \mathrm{Cl}$ & $P$ value $^{a}$ & $\overline{O R}$ & $95 \% \mathrm{Cl}$ & $P$ value $^{a}$ & $\overline{\mathrm{OR}}$ & $95 \% \mathrm{Cl}$ & $P$ value $^{a}$ \\
\hline Age (years) & 0.97 & $0.93,1.02$ & 0.241 & 0.97 & $0.93,1.03$ & 0.329 & 0.97 & $0.92,1.03$ & 0.312 \\
\hline \multicolumn{10}{|l|}{ Marital status } \\
\hline single & 1.0 & & & 1.0 & & & 1.0 & & \\
\hline cohabitation / married & 0.52 & $0.31,0.87$ & 0.012 & 0.47 & $0.27,0.81$ & 0.007 & 0.58 & $0.32,1.06$ & 0.076 \\
\hline divorced & 0.46 & $0.24,0.87$ & 0.017 & 0.46 & $0.23,0.92$ & 0.029 & 0.52 & $0.24,1.12$ & 0.097 \\
\hline widoved & 0.41 & $0.23,0.73$ & 0.003 & 0.40 & $0.21,0.76$ & 0.005 & 0.53 & $0.26,1.06$ & 0.073 \\
\hline \multicolumn{10}{|l|}{ Body mass index $\left(\mathrm{kg} / \mathrm{m}^{2}\right)$} \\
\hline$<25$ & 1.0 & & & 1.0 & & & 1.0 & & \\
\hline $25-29$ & 1.15 & $0.80,1.67$ & 0.450 & 1.31 & $0.89,1.93$ & 0.168 & 1.14 & $0.74,1.75$ & 0.562 \\
\hline$\geq 30$ & 1.43 & $0.96,2.11$ & 0.076 & 1.50 & $0.99,2.25$ & 0.054 & 0.87 & $0.55,1.38$ & 0.551 \\
\hline \multicolumn{10}{|l|}{ Alcohol consumption (g/month) } \\
\hline none & 1.0 & & & 1.0 & & & 1.0 & & \\
\hline$<360$ & 0.79 & $0.59,1.06$ & 0.115 & 0.84 & $0.61,1.16$ & 0.287 & 0.91 & $0.64,1.29$ & 0.593 \\
\hline$\geq 360$ & 0.45 & $0.19,1.05$ & 0.066 & 0.39 & $0.16,0.98$ & 0.045 & 0.34 & $0.12,0.97$ & 0.043 \\
\hline \multicolumn{10}{|l|}{ Life satisfaction } \\
\hline satisfied & 1.0 & & & 1.0 & & & 1.0 & & \\
\hline intermediate & 0.67 & $0.42,1.06$ & 0.084 & 0.61 & $0.37,1.01$ & 0.054 & 0.70 & $0.41,1.22$ & 0.212 \\
\hline dissatisfied & 0.71 & $0.44,1.17$ & 0.180 & 0.72 & $0.42,1.24$ & 0.235 & 0.74 & $0.41,1.34$ & 0.313 \\
\hline Number of prescribed medications (continuous) & 1.38 & $1.28,1.49$ & $<0.001$ & - & & & 1.37 & $1.25,1.50$ & $<0.001$ \\
\hline \multicolumn{10}{|l|}{ Amount of purchased antidepressants ${ }^{b}$} \\
\hline low & 1.0 & & & - & & & 1.0 & & \\
\hline moderate & 2.39 & $1.67,3.43$ & $<0.001$ & - & & & 2.56 & $1.68,3.89$ & $<0.001$ \\
\hline high & 4.28 & $2.95,6.20$ & $<0.001$ & - & & & 3.81 & $2.51,5.79$ & $<0.001$ \\
\hline \multicolumn{10}{|l|}{ History of work disability pension } \\
\hline no & 1.0 & & & - & & & 1.0 & & \\
\hline somatic cause & 0.85 & $0.61-1.18$ & 0.330 & - & & & 0.63 & $0.42,0.95$ & 0.026 \\
\hline psychiatric cause & 3.94 & $2.41-6.44$ & $<0.001$ & - & & & 2.55 & $1.46,4.46$ & 0.001 \\
\hline
\end{tabular}

Abbreviations: $\mathrm{Cl}$, confidence interval; $\mathrm{OR}$, odds ratio

a $P$ value from the chi-square test.

b Tertile cutoffs for amount of use (DDD/4 months): <33 (low), 33-99.9 (moderate), $\geq 100$ (high).

c Multivariate models include all the variables in the column. 
Table 5 Factors related to the sensitivity of self-reported use of any psychoactive medication compared to registerbased data $(n=1,638)$ by logistic models

\begin{tabular}{|c|c|c|c|c|c|c|c|c|c|}
\hline \multirow[b]{2}{*}{ Variables } & \multicolumn{3}{|c|}{ Univariate model } & \multicolumn{3}{|c|}{$\begin{array}{l}\text { Multivariate model } 1^{c} \\
(n=1,448)\end{array}$} & \multicolumn{3}{|c|}{$\begin{array}{l}\text { Multivariate model } 2^{c} \\
(n=1,446)\end{array}$} \\
\hline & OR & $95 \% \mathrm{Cl}$ & $P$ value $^{a}$ & $\mathrm{OR}$ & $95 \% \mathrm{Cl}$ & $P$ value $^{a}$ & $\overline{\mathrm{OR}}$ & $95 \% \mathrm{Cl}$ & $P$ value $^{a}$ \\
\hline Age (years) & 0.93 & $0.90,0.96$ & $<0.001$ & 0.93 & $0.90,0.97$ & $<0.001$ & 0.94 & $0.91,0.98$ & 0.006 \\
\hline \multicolumn{10}{|l|}{ Marital status } \\
\hline single & 1.0 & & & 1.0 & & & 1.0 & & \\
\hline cohabitation/married & 0.41 & $0.29,0.58$ & $<0.001$ & 0.42 & $0.29,0.62$ & $<0.001$ & 0.48 & $0.31,0.73$ & 0.001 \\
\hline divorced & 0.56 & $0.36,0.87$ & 0.009 & 0.55 & $0.34,0.89$ & 0.015 & 0.60 & $0.35,1.03$ & 0.064 \\
\hline widoved & 0.32 & $0.22,0.48$ & $<0.001$ & 0.34 & $0.22,0.53$ & $<0.001$ & 0.38 & $0.23,0.62$ & $<0.001$ \\
\hline \multicolumn{10}{|l|}{ Body mass index $\left(\mathrm{kg} / \mathrm{m}^{2}\right)$} \\
\hline$<25$ & 1.0 & & & 1.0 & & & 1.0 & & \\
\hline $25-29$ & 1.04 & $0.81,1.34$ & 0.751 & 1.15 & $0.88,1.49$ & 0.317 & 1.00 & $0.74,1.34$ & 0.989 \\
\hline$\geq 30$ & 1.42 & $1.09,1.86$ & 0.009 & 1.46 & $1.11,1.94$ & 0.008 & 0.95 & $0.69,1.31$ & 0.770 \\
\hline \multicolumn{10}{|l|}{ Alcohol consumption (g/month) } \\
\hline none & 1.0 & & & 1.0 & & & 1.0 & & \\
\hline$<360$ & 0.74 & $0.58,0.95$ & 0.018 & 0.68 & $0.55,0.85$ & 0.001 & 0.80 & $0.63,1.03$ & 0.079 \\
\hline$\geq 360$ & 0.63 & $0.49,0.81$ & $<0.000$ & 0.87 & $0.45,1.67$ & 0.672 & 0.89 & $0.42,1.87$ & 0.749 \\
\hline \multicolumn{10}{|l|}{ Life satisfaction } \\
\hline satisfied & 1.0 & & & 1.0 & & & 1.0 & & \\
\hline intermediate & 0.98 & $0.74,1.30$ & 0.880 & 0.99 & $0.73,1.35$ & 0.954 & 1.04 & $0.73,1.47$ & 0.837 \\
\hline dissatisfied & 1.36 & $1.00,1.87$ & 0.059 & 1.32 & $0.93,1.87$ & 0.118 & 1.20 & $0.81,1.78$ & 0.371 \\
\hline Number of prescribed medications (continuous) & 1.34 & $1.27,1.40$ & $<0.001$ & - & & & 1.32 & $1.25,1.40$ & $<0.001$ \\
\hline \multicolumn{10}{|l|}{ Amount of purchased psychoactive medication ${ }^{\text {b }}$} \\
\hline low & 1.0 & & & - & & & 1.0 & & \\
\hline moderate & 2.42 & $1.86,3.13$ & $<0.001$ & - & & & 1.85 & $1.38,2.47$ & $<0.001$ \\
\hline high & 6.65 & $5.05,8.75$ & $<0.001$ & - & & & 4.12 & $3.04,5.60$ & $<0.001$ \\
\hline \multicolumn{10}{|l|}{ History of work disability pension } \\
\hline no & 1.0 & & & - & & & 1.0 & & \\
\hline somatic cause & 1.02 & $0.81,1.27$ & 0.889 & - & & & 0.66 & $0.50,0.86$ & 0.003 \\
\hline psychiatric cause & 10.49 & $6.70,16.42$ & $<0.001$ & - & & & 6.53 & $3.90,10.92$ & $<0.001$ \\
\hline
\end{tabular}

Abbreviations: $\mathrm{Cl}$, confidence interval; $O R$, odds ratio.

${ }^{a} P$ value from the chi-square test.

b Tertile cutoffs for amount of use (DDD/4 months): <34 (low), 34-100 (moderate), >100 (high).

c Multivariate models include all the variables in the column.

Also among women with register-based sole antidepressants use $(\mathrm{n}=393)$, a greater number of any prescribed medication $(\mathrm{OR}=1.33, P<0.001)$, amount of purchased antidepressants $\left(\mathrm{OR}_{\text {moderate use }}=2.89, P<0.001\right.$ and $\mathrm{OR}_{\text {high }}$ use $=3.57, P<0.001)$ and history of work disability due to psychiatric cause $(\mathrm{OR}=4.04, P=0.002)$ remained correlates of self-report in the multivariate model 2 (Cf. Table 4).

\section{Loss analysis}

According to the prescription register, the 1,025 women who did not return the questionnaire, purchased (during the four months preceding the follow-up enquiry in 1999) more often than the responders who reported any psychoactive medication $(23.3$ vs. $14.8 \%, P<0.001)$, antidepressants (9.3 vs. $7.0 \%, P=0.010$ ) or other psychoactive medications ( 14.0 vs. $7.8 \%, P<0.001)$. The corresponding proportions, for the 506 women who returned the questionnaire but did not answer the medication question were similar with those of the responders (15.2, 6.3 and $8.9 \%$, respectively). Correction of non-response to the enquiry or the medication question increased the population estimate for use of any psychoactive medication from 14.8 to $15.5 \%$, use of antidepressants from 7.0 to $7.2 \%$ and use of other psychoactive medication from 7.8 to $8.4 \%$. 


\section{Discussion}

Postmenopausal women are at increased risk of depression. This study investigated agreement between self-reported use of psychoactive medication and the National prescription register data and possible related factors among postmenopausal women. The sensitivity of self-report was $44 \%$ for any psychoactive medication and 55\% for antidepressants, while it was over $80 \%$ for diuretics. However, higher use and history of work disability pension due to psychiatric cause increased sensitivity strongly.

Methodological differences such as selected golden standard, interview vs. enquiry method or structure and wording of questions might be reasons for the differences in the results between studies. Other Finnish studies have previously found high overall agreement (ad 0.87 ) for antidepressants $[18,19]$ and antipsychotics $[18,19,22]$ with the same golden standard as in this study. This agreement difference might be due to longer purchase time window $[18,22]$, younger population $[18,22]$ or the interview vs. enquiry method and prescriptions brought to the interview [18,19]. A large study by Nielsen et al. [13] with home interview but pharmacy records as a reference, found self-report sensitivity of antidepressant use (56\%) similar to ours. Also Boudreau et al. [9] found lower sensitivity for antidepressants (4466\%) when using interview with specific medication questions. However, higher sensitivities for self-report of antidepressants and psychoactive medication (70-89\%) have generally been obtained with different methodologies; such as medical records [12] or physician-reported data as golden standard [16], when asked "never/ever" use [16] or a direct question on medication use [17,21] or when prescriptions were brought to the interview [18]. On the other hand, Nielsen et al. [13] also found low sensitivity for psycholeptics (38\%) and especially for hypnotics and sedatives (27\%). Thus, the low sensitivity in our study (29\%) for the group of other psychoactive medication might be due to the high proportion of users of hypnotics and sedatives in our study sample. Similar to our study, moderate overall agreement (>0.50) for psycholeptics [13] (vs. psychoactive medication) and even better for antidepressants reporting $[12,13,16,17,22]$ has been shown by others.

In the present study, users of psychoactive medication showed lower sensitivity for diuretics than nonusers, but they reported the use of diuretics better than the use of psychoactive medication. Also in previous studies, the use of psychoactive medication has been less accurately reported than other medications $[9,11,13]$. Interestingly, however, a lower sensitivity in respect to psychoactive medication in this study was linearly related to less severe psychiatric problems (i.e. no work disability pension due to psychiatric cause) and lower use of psychoactive medication. One explanation may simply be that psychoactive medicines such as hypnotics are not used as regularly as diuretics. One might just not feel that occasional use is important enough to be mentioned in a questionnaire or the medicine may not be used at the moment or at all after purchase. Lin et al. [32] found that $28 \%$ of patients discontinued their antidepressant medication during the first month of therapy and $44 \%$ by the third month of therapy. Longer and regular use may increase recall or reduce self-perceived shame or unwillingness to report the use $[10,11,16,33]$.

In Finland the use of antidepressants doubled between 1999-2009 [34], which might decrease the unwillingness to report use of medication. However, our study subjects increased less their use of antidepressants between 1999-2009 and the sensitivity of self-report of antidepressants in 2009 was similar to that in 1999 (data not shown). It seems that regular use of antidepressants and/or long-term severe mental illness (as indicated by work disability pension) are the most important correlates of sufficient selfreport.

In general, several factors may affect the agreement between self-reports and register-based data. Obesity has been previously positively associated with agreement when self-reports have been compared to medical records [12], but higher BMI is associated also with depression [35]. Thus, after including the amount of antidepressants or psychoactive medication (model 2), the significance of BMI in respect to sensitivity disappeared. Lower income $[5,16]$ and education level $[3,5,10,22]$ have been associated with poorer agreement, but Sjahid et al. [6] found no differences according to educational or socio-economic status. Homogeneity of our cohort may have played a role in some of the factors. In our female sample, only a small number of women had a higher education, which may have contributed to the nonsignificance of education. Older age has $[11,36]$ or has not [6] been associated with decreased validity of self-reports, while narrow age window in our study (57-68 years) might explain the found minimal age effect. In this study, being single was associated with the highest sensitivity in reporting psychoactive medication use, while in another Finnish cohort born in 1966 [22] the same was true for the married subjects. Lastly, even if Van den Brandt et al. [11] study suggested a decrease in recall along with increasing number of prescribed medications, in our study higher number of any prescribed medication currently in use was associated with better sensitivity. Stability of disease status indicated by history of work disability pension and amount of psychoactive 
medication in our study seemed to be more important positive determinants of agreement than putative (negative) effects of mental disease or its medication.

The strengths of this study include a large populationbased cohort, high response rate and utilization of the National prescription register, which has a coverage of 97\% and ability to detect also slight (i.e. irregular) use [31]. The narrow age range increased the homogeneity of the sample, but provides data on important period around retirement. Lastly, our results apply to postmenopausal women, a risk group for depression, and a base for future growing elderly population. Our method of asking current use of any prescribed medications may give lower agreement when compared to the results obtained by never-ever -type questions. Since the prescription register as a reference indicates only the medication purchased and not the actual use, it possesses a possibility to non-agreement, but this inaccuracy was minimized by checking also purchase of medication after enquiry.

\section{Conclusions}

Less than half of the women reported their use of any psychoactive medication and just over half their use of antidepressants compared to register-based data on purchased medication. The sensitivity of self-report increased considerably with higher use, with a history of work disability pension due to psychiatric cause or when occasional use was excluded. In conclusion, for research purposes, self-reported current medication use seems in general to be a sufficient indicator of regular use of antidepressants or in respect of use of any psychoactive medication, for those with severe psychiatric disease. However, prescription register data is more reliable for those using these medications irregularly and with less severe mental disorders.

\section{Ethical standards}

This study was approved by the ethics committee of Kuopio University Hospital, Finland.

\section{Abbreviations Study; SD: Standard deviation. \\ Competing interests \\ All authors declare that they have no conflicts of interest.}

ATC: Anatomical-Therapeutic-Chemical; BMI: Body mass index; Cl: Confidence interval; DDD: Defined daily doses; HT: Hormone therapy; LS: Life satisfaction; OR: Odds ratio; OSTPRE: Kuopio Osteoporosis Risk Factor and Prevention

\section{Authors' contributions}

PR, HK-H and RH designed the study. PR conducted data analysis, data interpretation, and the writing of the manuscript. $\mathrm{HK}-\mathrm{H}, \mathrm{RH}$ and JK were responsible for the data and contributed to data interpretation. $\mathrm{RH}, \mathrm{MT}$ and HK were responsible for the original OSTPRE study design. All the authors contributed to the revision of the manuscript and approved the final version.

\section{Acknowledgements}

Research has been supported by the strategic of the University of Eastern Finland, by an EVO grant of Lapland Hospital District (to HK-H, PR and RH) and by Academy of Finland (grants 115969 and 250707; to HK). The authors thank Finnish Medicines Agency (FIMEA) and Social Insurance Institution of Finland (KELA). We would also like to thank Prof. Riitta Ahonen for initial ideas, Dr. Lana Williams for linguistic check and Ms. Seija Oinonen and MSc Kimmo Ronkainen for technical help.

\section{Author details}

${ }^{1}$ Social Pharmacy, School of Pharmacy, Faculty of Health Sciences, University of Eastern Finland (UEF), P.O. Box 1627, 70211, Kuopio, Finland. ${ }^{2}$ Bone and Cartilage Research Unit, Surgery, Institute of Clinical Medicine, UEF, Kuopio, Finland. ${ }^{3}$ Institute of Clinical Medicine, Psychiatry, UEF, Kuopio, Finland. ${ }^{4}$ Institute of Clinical Medicine, Psychiatry, University of Oulu, Oulu, Finland. ${ }^{5}$ Departments of Psychiatry; Kuopio University Hospital (KUH), Kuopio; SouthSavonia Hospital District, Mikkeli; North Karelia Central Hospital, Joensuu; SOSTERI, Savonlinna; SOTE, lisalmi; Lapland Hospital District, Rovaniemi, Finland. 'Department of Orthopaedics, Traumatology and Hand Surgery, KUH, Kuopio, Finland. 'D Department of Obstetrics and Gynaecology, KUH, Kuopio, Finland. ${ }^{8}$ Institute of Public Health and Clinical Nutrition, UEF, Kuopio, Finland.

Received: 4 March 2013 Accepted: 24 June 2013

Published: 2 July 2013

\section{References}

1. Jain MG, Rohan TE, Howe GR: Agreement of self-reported use of menopausal hormone replacement therapy with physician reports. Epidemiology 1999, 10(3):260-263.

2. Banks E, Beral V, Cameron R, Hogg A, Langley N, Barnes I, Bull D, Elliman J, Harris CL: Agreement between general practice prescription data and self-reported use of hormone replacement therapy and treatment for various illnesses. J Epidemiol Biostat 2001, 6(4):357-363.

3. Sandini L, Pentti K, Tuppurainen M, Kröger H, Honkanen R: Agreement of self-reported estrogen use with prescription data: an analysis of women from the Kuopio Osteoporosis Risk Factor and Prevention Study (OSTPRE). Menopause 2008, 15(2):282-289.

4. Norell SE, Boethius G, Persson I: Oral contraceptive use: interview data versus pharmacy records. Int J Epidemio/ 1998, 27(6):1033-1037.

5. Curtis JR, Westfall AO, Allison J, Freeman A, Kovac SH, Saag KG: Agreement and validity of pharmacy data versus self-report for use of osteoporosis medications among chronic glucocorticoid users. Pharmacoepidemiol Drug Saf 2006, 15(10):710-718.

6. Sjahid SI, van der Linden PD, Stricker BH: Agreement between the pharmacy medication history and patient interview for cardiovascular drugs: the Rotterdam elderly study. Br J Clin Pharmacol 1998, 45(6):591-595.

7. Smith NL, Psaty BM, Heckbert SR, Tracy RP, Cornell ES: The reliability of medication inventory methods compared to serum levels of cardiovascular drugs in the elderly. J Clin Epidemiol 1999, 52(2):143-146.

8. Klungel $\mathrm{OH}$, de Boer $\mathrm{A}$, Paes $\mathrm{AH}$, Herings RM, Seidell JC, Bakker A: Agreement between self-reported antihypertensive drug use and pharmacy records in a population-based study in the Netherlands. Pharm World Sci 1999, 21(5):217-220.

9. Boudreau DM, Daling JR, Malone KE, Gardner JS, Blough DK, Heckbert SR: A validation study of patient interview data and pharmacy records for antihypertensive, statin and antidepressant medication use among older women. Am J Epidemiol 2004, 159(3):308-317.

10. Klungel $\mathrm{OH}$, de Boer A, Paes AHP, Herings RMC, Seidell JC, Bakker A: Influence of question structure on the recall of self-reported drug use. J Clin Epidemiol 2000, 53(3):273-277.

11. Van den Brandt PA, Petri H, Dorant E, Goldbohm RA, Van de Crommert S: Comparison of questionnaire information and pharmacy data on drug use. Pharm Weekb/ Sci 1991, 13(2):91-96.

12. Tisnado DM, Adams JL, Liu H, Damberg CL, Chen W-P, Hu FA, Carlisle DM, Mangione CM, Kahn KL: What is the concordance between the medical record and patient self-report as data sources for ambulatory care? Med Care 2006, 44(2):132-140. 
13. Nielsen MW, Søndergaard B, Kjøller M, Hansen EH: Agreement between self-reported data on medicine use and prescription records vary according to method of analysis and therapeutic group. $J$ Clin Epidemiol 2008, 61(9):919-924.

14. World Health Organization: The Global Burden of Disease: 2004 Update. Geneva, Switzerland: WHO Press; 2008.

15. Eaton WW, Anthony JC, Gallo J, Cai G, Tien A, Romanoski A, Lyketsos C, Chen LS: Natural history of Diagnostic Interview Schedule/DSM-IV major depression. The Baltimore Epidemiologic Catchment Area follow-up. Arch Gen Psychiatry 1997, 54(11):993-999.

16. Cotterchio M, Kreiger N, Darlington G, Steingart A: Comparison of selfreported and physician-reported antidepressant medication use. Ann Epidemiol 1999, 9(5):283-289.

17. Kwon A, Bungay KM, Pei Y, Rogers WH, Wilson IB, Zhou Q, Adler DA Antidepressant use: concordance between self-report and claims records. Med Care 2003, 41(3):368-374.

18. Haukka J, Suvisaari J, Tuulio-Henriksson A, Lönnqvist J: High concordance between self-reported medication and official prescription database information. Eur J Clin Pharmacol 2007, 63(11):1069-1074.

19. Rikala M, Hartikainen S, Sulkava R, Korhonen MJ: Validity of the Finnish Prescription Register for measuring psychotropic drug exposures among elderly finns: a population-based intervention study. Drugs Aging 2010, 27(4):337-349.

20. Gilchrist WJ, Lee YC, Tam HC, Macdonald JB, Williams BO: Prospective study of drug reporting by general practitioners for an elderly population referred to a geriatric service. Br Med J (Clin Res Ed) 1987, 294(6567):289-290.

21. Pit SW, Byles JE, Cockburn J: Accuracy of telephone self-report of drug use in older people and agreement with pharmaceutical claims data. Drugs Aging 2008, 25(1):71-80.

22. Haapea M, Miettunen J, Lindeman S, Joukamaa M, Koponen H: Agreement between self-reported and pharmacy data on medication use in the Northern Finland 1966 Birth Cohort. Int J Methods Psychiatr Res 2010, 19(2):88-96.

23. Tuppurainen M, Honkanen R, Kröger H, Saarikoski S, Alhava E: Osteoporosis risk factors, gynaecological history and fractures in perimenopausal women - The results of the baseline postal enquiry of the Kuopio Osteoporosis Risk Factor and Prevention Study. Maturitas 1993, 17(2):89-100.

24. Saarelainen J, Kiviniemi V, Kröger H, Tuppurainen M, Niskanen L, Jurvelin J, Honkanen R: Body mass index and bone loss among postmenopausal women: the 10-year follow-up of the OSTPRE cohort. J Bone Miner Metab 2012, 30(2):208-216.

25. Koivumaa-Honkanen $\mathrm{H}$, Honkanen R, Viinamäki H, Heikkilä K, Kaprio J, Koskenvuo M: Self-reported life satisfaction and 20-year mortality in healthy Finish adults. Am J Epidemiol 2000, 152(10):983-991.

26. Koivumaa-Honkanen $\mathrm{H}$, Honkanen R, Viinamäki H, Heikkilä K, Kaprio J, Koskenvuo M: Life satisfaction and suicide, a 20-year follow-up study. Am J Psychiatry 2001, 158(3):433-439.

27. Koivumaa-Honkanen $H$, Kaprio J, Honkanen R, Viinamäki H, Koskenvuo M: Life satisfaction and depression in a 15-year follow-up of healthy adults. Soc Psychiatry Psychiatr Epidemiol 2004, 39(12):994-999.

28. Rissanen T, Viinamäki H, Honkalampi K, Lehto SM, Hintikka J, Saharinen T, Koivumaa-Honkanen $\mathrm{H}$ : Long term life dissatisfaction and subsequent major depressive disorder and poor mental health. BMC Psychiatry 2011, 23(11):140-146.

29. Paunio T, Korhonen T, Hublin C: Longitudinal study on poor sleep and life dissatisfaction in a nationwide cohort of twins. Am J Epidemiol 2009, 169(2):206-213.

30. World Health Organization: WHO Collaborating Centre for Drug Statistic Methodology, Guidelines for ATC classification and DDD assignment 2010. Oslo: World Health Organization; 2009

31. Peura S, Rajaniemi S, Kurkijärvi U: Pharmaceutical Pricing and Reimbursement Information Project: Finland 2007. 2007. http://whocc.goeg.at/Literaturliste/ Dokumente/CountryInformationReports/Finland_PPRI_2007.pdf.

32. Lin EH, Von Korff M, Katon W, Bush T, Simon GE, Walker E, Robinson P: The role of the primary care physician in patients' adherence to antidepressant therapy. Med Care 1995, 33(1):67-74.

33. Goldman LS, Nielsen NH, Champion HC: Awareness, diagnosis and treatment of depression. J Gen Intern Med 1999, 14:569-580.
34. Finnish Medicines Agency Fimea and Social Insurance Institution: Finnish statistics on medicines 2010. Helsinki: Edita Prima Oy; 2011.

35. Luppino FS, de Wit LM, Bouvy PF, Stijnen T, Cuijpers P, Penninx BWJH, Zitman FG: Overweight, obesity and depression. A systematic review and meta-analysis of longitudinal studies. Arch Gen Psychiatry 2010, 67(3):220-229.

36. West SL, Savitz DA, Koch G, Strom BL, Guess HA, Hartzema A: Recall accuracy for prescription medications: Self-report compared with database information. Am J Epidemiol 1995, 142(10):1103-1112.

doi:10.1186/1471-244X-13-180

Cite this article as: Rauma et al:: The relationship between self-reported and registry-based data on use of psychoactive medications in postmenopausal women. BMC Psychiatry 2013 13:180.

\section{Submit your next manuscript to BioMed Central and take full advantage of:}

- Convenient online submission

- Thorough peer review

- No space constraints or color figure charges

- Immediate publication on acceptance

- Inclusion in PubMed, CAS, Scopus and Google Scholar

- Research which is freely available for redistribution

Submit your manuscript at www.biomedcentral.com/submit
C) BioMed Central 